\title{
A novel rituximab administration protocol to minimize infusion-related adverse reactions in patients with B-cell non-Hodgkin lymphoma
}

\section{Daisuke Tsutsumi}

Nihon University: Nihon Daigaku

\section{Tatsuya Hayama}

Nihon University Itabashi Hospital: Nihon Daigaku Igakubu Fuzoku Itabashi Byoin

Katsuhiro Miura ( $\nabla$ miura.katsuhiro@nihon-u.ac.jp)

Nihon University https://orcid.org/0000-0002-1538-3804

\section{Akihiro Uchiike}

Nihon University Itabashi Hospital: Nihon Daigaku Igakubu Fuzoku Itabashi Byoin

\section{Shinya Tsuboi}

Nihon University Itabashi Hospital: Nihon Daigaku Igakubu Fuzoku Itabashi Byoin

\section{Susumu Otsuka}

Nihon University Itabashi Hospital: Nihon Daigaku Igakubu Fuzoku Itabashi Byoin

\section{Yoshihiro Hatta}

Nihon University: Nihon Daigaku

\section{Yukinaga Kishikawa}

Nihon University: Nihon Daigaku

\section{Research Article}

Keywords: B-cell non-Hodgkin lymphoma. Bulky disease, Rituximab, Indolent histology, nfusion-related reaction

Posted Date: September 17th, 2021

DOl: https://doi.org/10.21203/rs.3.rs-909741/v1

License: (c) (i) This work is licensed under a Creative Commons Attribution 4.0 International License. Read Full License 


\section{Abstract \\ Background}

Rituximab is widely used as a key component of immunochemotherapy to treat B-cell non-Hodgkin lymphoma (B-NHL). However, infusion-related reactions (IRRs) during drug administration are occasionally severe or even life-threatening and thus remain problematic for patients and healthcare providers.

\section{Aim}

To minimize IRRs to rituximab in patients with various types of B-NHL.

\section{Method:}

We stratified patients into low- $(n=39)$, moderate- $(n=35)$, and high-risk $(n=7)$ groups according to the number of risk factors, specifically, an indolent histology and the presence of bulky tumors $(>10 \mathrm{~cm})$. For the first rituximab cycle, the low- and moderate-risk groups underwent conventional infusion \#1 ( 4.3 h), and the high-risk group underwent long infusion ( $6.8 \mathrm{~h})$. Patients in the low-, moderate-, and high-risk groups without IRRs in the first cycle underwent short infusion $(2.3 \mathrm{~h})$, conventional infusion \#2 (3.5 h), and conventional infusion \#1, respectively. Patients with IRRs in the first cycle received a second rituximab cycle with the same schedule as that in the first cycle. The procedure for the third cycle was at the attending physician's discretion.

\section{Results}

Among 81 B-NHL patients, the incidences of IRRs in the low-, moderate-, and high-risk groups were $31 \%$, $20 \%$, and $57 \%$, respectively, without any grade $\geq 3$ IRRs. The overall conversion rate to short infusion in the third cycle was $54 \%$, without any IRRs.

\section{Conclusion}

Our step-by-step protocol provided safe and comfortable rituximab administration for both patients and practitioners (UMIN-CTR; UMIN000032309, registered on 19th April 2018).

\section{Impact Of Findings On Practice}

- The first administration of rituximab in patients with B-cell non-Hodgkin lymphoma (B-NHL) is usually challenging because infusion-related reactions (IRRs) to the drug are anticipated the most in 
the initial cycle.

- IRRs to rituximab in the following cycle are less common; however, how to minimize the incidence and severity of IRRs remains unclear.

- This study demonstrated that the risk-stratified, step-by-step protocol of rituximab infusion might represent a safe rituximab administration method in patients with various types of B-NHL.

- The developed rituximab infusion protocol could help avoid unexpected, severe IRRs and medical expenses.

\section{Introduction}

Previous studies have demonstrated the clinical efficacy of rituximab, a chimeric anti-CD20 monoclonal antibody, in treating B-cell non-Hodgkin lymphoma (B-NHL). Currently, rituximab is administered as part of the standard treatment for various subtypes of $\mathrm{B}-\mathrm{NHL}$ [1]. Although the toxicity profile of rituximab is generally favorable, infusion-related reactions (IRRs), such as fever, chills, nausea, rashes, rigors, and hypotension, are a major concern. These reactions usually develop immediately after rituximab infusion but can occur within $24 \mathrm{~h}$ after drug administration. They are likely caused by cytokines rather than allergy-related events, but the exact mechanism remains unclear [2].

The incidence of IRRs to rituximab varies across studies; however, more than half of the patients in previous studies experienced IRRs, particularly during the initial infusion of rituximab [3]. Most IRRs are mild to moderate in severity and may be managed by suspending drug infusion and corticosteroid administration [4]. However, treatment interruption associated with these interventions increases the burden on patients and healthcare providers. While a slower rituximab infusion may reduce the need for treatment interruption for IRRs, prolonged total infusion time interferes with the operations of busy outpatient chemotherapy centers such as ours. Therefore, there is a trade-off between reducing the risk of IRRs and shortening the administration time of rituximab, which has been a problematic issue in anticancer treatment for B-NHL. In addition, IRRs to rituximab are severe or life-threatening in approximately $10 \%$ of patients $[5,6]$. Therefore, quantifying the risk of IRRs is a major concern for oncology practitioners.

In a previous study, we demonstrated that low-grade histology and bulky disease with a tumor diameter > $10 \mathrm{~cm}$ are independent risk factors for IRRs to rituximab [7]. However, to the best of our knowledge, no prospective or retrospective studies have evaluated a novel protocol that can minimize IRRs to rituximab among B-NHL patients with various risk factors. Therefore, we developed a protocol for rituximab administration utilizing these risk factors with the aim of preventing severe IRRs during a shorter infusion period at the outpatient chemotherapy center of the Nihon University Itabashi Hospital. In addition, we designed a prospective observational study that aimed to verify the suitability of our rituximab dosing schedule. Herein, we report our findings on the utility of our rituximab infusion protocol, which involved a simple combination of risk factors for IRRs. 
This study aimed to establish an effective procedure for safely administering rituximab within a short time for patients with various B-NHL subtypes, utilizing our previously identified risk factors for IRR as a stratification tool.

\section{Ethics approval}

This study was conducted in accordance with the Declaration of Helsinki. All patients provided written informed consent for participation in the study, along with their consent for treatment with rituximab. This study, including data collection, analysis, and publication, was approved by the Nihon University Itabashi Hospital Clinical Research Judging Committee on 23rd March 2018 (RK-180313-1). In addition, the study was registered in a Japanese database for clinical studies on 19th April 2018 (UMIN-CTR; UMIN000032309).

\section{Method}

\section{Patients}

B-NHL patients aged $\geq 20$ years and treated with rituximab at the Nihon University Hospital from April 2018 to November 2019 were enrolled in this study after obtaining their written informed consent. Patients with a history of rituximab treatment before April 2018 were excluded.

\section{Preparation and premedication}

Rituximab was diluted with saline to a final concentration of $1 \mathrm{mg} / \mathrm{mL}$ and intravenously administered to patients at a dose of $375 \mathrm{mg} / \mathrm{m}^{2}$. Approximately 30 minutes before rituximab administration, all patients received $400 \mathrm{mg}$ of oral acetaminophen and $2 \mathrm{mg}$ of chlorpheniramine maleate as prophylactic premedication.

\section{Administration of rituximab}

We stratified patients into low-, moderate-, and high-risk groups according to the observed number of risk factors $(0,1$, and 2 risk factors, respectively) identified in our previous study, specifically, an indolent histology and the presence of a bulky disease with a tumor diameter $>10 \mathrm{~cm}$ [7]. Following this stratification, we established a rituximab dosing regimen for the first and second cycles. The infusion procedure in the third cycle was at the attending physician's discretion since most IRRs occur during the initial rituximab administration and the incidence of IRRs is known to decrease in subsequent cycles [7, 8].

The first rituximab cycle was administered according to the package insert in principle and provided to each group as follows: In the low-risk group, the infusion rate of rituximab was set at $25 \mathrm{mg} / \mathrm{h}$ for the first hour, $100 \mathrm{mg} / \mathrm{h}$ for the next hour, and a maximum of $200 \mathrm{mg} / \mathrm{h}$ thereafter (conventional infusion \#1, approximately $4.3 \mathrm{~h}$ for a total infusion in a patient with a body surface area [BSA] of $1.6 \mathrm{~m}^{2}$ ); no additional prophylactic medication was administered. In the moderate-risk group, rituximab was 
administered similar to that in the low-risk group, but an additional prophylactic dose of $300 \mathrm{mg}$ hydrocortisone phosphate was administered 30 minutes before rituximab administration. In the high-risk group, the rituximab infusion rate was set at $25 \mathrm{mg} / \mathrm{h}$ for the first hour and limited at a maximum of 100 $\mathrm{mg} / \mathrm{h}$ thereafter due to the risk of severe IRRs (long infusion, approximately $6.8 \mathrm{~h}$ for a total infusion in a patient with a BSA of $1.6 \mathrm{~m}^{2}$ ); an additional prophylactic dose of $300 \mathrm{mg}$ hydrocortisone phosphate was administered.

The second cycle of rituximab infusion was dependent on the occurrence of IRRs in the first cycle. If a patient in the low-risk group did not experience IRRs to rituximab in the first cycle, he/she received the second cycle of rituximab as follows: a prophylactic dose of $300 \mathrm{mg}$ hydrocortisone phosphate was administered, and the rituximab infusion was started at $100 \mathrm{mg} / \mathrm{h}$ for the first 30 minutes and increased by $100 \mathrm{mg} / \mathrm{h}$ every 30 minutes up to a maximum of $400 \mathrm{mg} / \mathrm{h}$ (short infusion, approximately $2.3 \mathrm{~h}$ for a total infusion in a patient with a BSA of $1.6 \mathrm{~m}^{2}$ ). Patients in the moderate-risk group without any IRRs in the first cycle received the second cycle of rituximab at an infusion rate of $100 \mathrm{mg} / \mathrm{h}$ for the first hour and a maximum of $200 \mathrm{mg} / \mathrm{h}$ thereafter (conventional infusion \#2, approximately $3.5 \mathrm{~h}$ for a total infusion in a patient with a BSA of $1.6 \mathrm{~m}^{2}$ ). Patients in the high-risk group who did not experience any IRR in the first cycle received the second rituximab as per the conventional infusion \#1. For all three groups, if a patient experienced any grade of IRR in the first cycle, the second cycle of rituximab was administered on the same dosing and schedule as the first. The infusion procedure for the third cycle was at the discretion of the attending physicians. The workflow of rituximab administration is shown in Fig. 1.

\section{Definition of IRRs}

IRRs were defined as adverse reactions to rituximab infusion, including rash, urticaria, angioedema, flushing, nausea, abdominal pain, nasal congestion, bronchospasm, hypotension, hypoxia, myocardial infarction, cardiovascular reactions, anaphylactoid events, and death. Each IRR was graded according to the National Cancer Institute Common Terminology Criteria for Adverse Events, version 4 (https://evs.nci.nih.gov/ftp1/CTCAE/CTCAE_4.03/, accessed on 10th September 2021).

\section{Statistical analysis}

The primary outcome was the incidence of IRRs to the first and second cycles of rituximab in each risk group. The secondary outcomes were the overall incidence of IRRs to the first and second cycles of rituximab, the infusion rates of rituximab during the onset of an IRR, the conversion rate to short infusion at the third cycle in each group, and the overall conversion rate to short infusion at the third cycle. We set the target number of patients as 70 to validate IRR incidence in each risk group; this was half the number selected for our previous study, in which approximately $40 \%$ of the patients experienced IRRs to rituximab [7]. The sample size was equivalent to the minimum required to detect a $30 \%$ reduction in IRR incidence in all patients with $80 \%$ power and $10 \%$ alpha error based on a one-sided test. Continuous variables are presented as means or medians, as appropriate, while categorical variables are presented as counts and percentages. Intergroup differences were compared using the $\chi^{2}$ test for categorical variables. Statistical 
significance was set at $p<0.05$. All statistical analyses were performed using JMP version 14.3 (SAS Institute, Cary, NC, USA).

\section{Results}

Eighty-three patients were registered, but two patients were excluded from the analysis because they did not receive rituximab after consenting to the study due to disease severity or a change in the treatment plan to radiation therapy. The remaining 81 patients with a median age of 69 years (range, 22-87 years) and a mean BSA of $1.63 \mathrm{~m}^{2}$ were included in the study. The most common disease subtype was diffuse large B-cell lymphoma (62\%), followed by follicular lymphoma (11\%). The most common chemotherapy regimen combined with rituximab was cyclophosphamide, doxorubicin, vincristine, and prednisolone (73\%), followed by bendamustine (21\%). In 67 (83\%) patients, rituximab was preceded by cytotoxic chemotherapy during the initial cycle of immunochemotherapy. Thirty-nine (48\%), 35 (43\%), and 7 (9\%) patients were stratified into the low-, moderate-, and high-risk groups, respectively (Table 1). 
Table 1

Patient characteristics

\begin{tabular}{|ll|}
\hline Characteristic & Value \\
\hline Total number of patients & 81 \\
\hline Gender, N (\%) & $52(64)$ \\
\hline Men & $29(36)$ \\
\hline Women & $69(60-77)$ \\
\hline Median age (range), years & \\
\hline Histological subtypes, N (\%) & $22(27)$ \\
\hline Indolent lymphoma ${ }^{a}$ & $59(73)$ \\
\hline Aggressive lymphoma & $27(33)$ \\
\hline Bulky disease $(>10 \mathrm{~cm})$ & $216[169-312]$ \\
\hline Median LDH $[$ [IQR], U/mL & $0.86[0.31-1.24]$ \\
\hline Median ALC [IQR], $\times 10^{9} / \mathrm{L}$ & \\
\hline
\end{tabular}

IQR, inter-quartile range; $L D H$, lactate dehydrogenase; $A L C$, absolute lymphocyte count

a Indolent lymphomas included follicular lymphoma grade 1/2, mucosa-associated lymphoid tissue lymphoma, splenic marginal zone lymphoma, and Waldenström's macroglobulinemia/lymphoplasmacytic lymphoma

${ }^{b}$ Aggressive lymphomas included Burkitt lymphoma, mantle cell lymphoma, follicular lymphoma (grade 3B), primary mediastinal large B-cell lymphoma, and diffuse large B-cell lymphoma

${ }^{\mathrm{c}}$ Normal upper limit of $220 \mathrm{U} / \mathrm{mL}$

\section{IRRs to rituximab}

After the first cycle of rituximab administration, IRRs were observed in 12, 7, and 4 patients in the lowmoderate-, and high-risk groups, respectively. Grade 2 IRRs were observed in 8, 4, and 4 patients in the low-, moderate-, and high-risk groups, respectively. Patients with grade 2 IRRs should be observed for at least approximately half an hour to assess their condition and whether additional medications should be prescribed or the infusion rate adjusted. Rituximab infusion was restarted after the symptoms disappeared in all patients with IRRs. Accordingly, the planned cycles of rituximab were successfully administered to all patients.

All 81 patients received a second cycle of rituximab. Among them, 27 patients in the low-risk group received a second dose of rituximab by short infusion according to the study protocol. In the second 
cycle, IRR to rituximab occurred in only 1 (1\%) patient, and this IRR was of grade 1 severity. This patient belonged to the low-risk group and experienced a second IRR after receiving conventional infusion \#1. During the first and the second cycles of rituximab, the prophylactic regimen and infusion rate were modified in 2 and 3 patients, respectively. Therefore, the overall compliance rate for the study protocol was $94 \%$.

After the completion of two cycles of therapy, 80 patients proceeded to the third cycle. Of these, 32,10 , and 2 patients in the low-, moderate-, and high-risk groups, respectively, received the third dose of rituximab by short infusion. No IRRs were observed during this cycle (Fig. 2).

The incidence of IRRs during the first and the third cycle in the low-, moderate-, and high-risk groups were $31 \%, 20 \%$, and $57 \%$, respectively, with no significant difference among the three risk groups $\left(\chi^{2}=3.923, p\right.$ $=0.1407)$. The overall incidence of IRR during the first and third cycles was $28 \%$, with no observed severity of grade 3 or higher. IRRs occurred most frequently among patients who received rituximab at an infusion rate of $100 \mathrm{mg} / \mathrm{h}$ (Fig. 3). The conversion rates to short infusion at the third rituximab cycle were $82 \%, 29 \%$, and $29 \%$ in the low-, moderate-, and high-risk groups, respectively, with a significant difference among the groups $\left(X^{2}=24.772, p<0.0001\right)$. The overall conversion rate to short infusion in the third cycle of rituximab among the entire cohort was $54 \%$ (Table 2 ).

Table 2

Incidence of infusion-related reactions and conversion rate to short infusion in each risk group

\begin{tabular}{|llllll|}
\hline & $\begin{array}{l}\text { Low-risk } \\
\text { group } \\
(\mathbf{N}=39)\end{array}$ & $\begin{array}{l}\text { Moderate-risk } \\
\text { group } \\
(\mathbf{N}=\mathbf{3 5})\end{array}$ & $\begin{array}{l}\text { High-risk } \\
\text { group } \\
\mathbf{( N = 7 )}\end{array}$ & $\begin{array}{l}\text { Total } \\
\mathbf{( N = 8 1 )}\end{array}$ & P-value \\
\hline IRR during cycle \#1 & $12(31 \%)$ & $7(20 \%)$ & $4(57 \%)$ & $\begin{array}{l}23 \\
(28 \%)\end{array}$ & 0.1407 \\
\hline IRR during cycle \#2 & $1(3 \%)$ & 0 & 0 & 0 & NA \\
\hline $\begin{array}{l}\text { Short infusion on cycle } \\
\# 3\end{array}$ & $32(82 \%)$ & $10(29 \%)$ & $2(29 \%)$ & 44 & $<$ \\
\hline IRR during cycle \#3 & 0 & 0 & & 0 & NA \\
\hline IRR, infusion-related reaction; NA, not applicable & & & 0.0001 \\
\hline
\end{tabular}

\section{Discussion}

In this study, our step-by-step procedure allowed the safe administration of rituximab, providing comfort for both patients and oncology practitioners. Remarkably, only $1 \%$ of the patients in this study experienced IRRs in the second rituximab cycle, and no patient experienced IRRs in the third rituximab cycle; however, $33 \%$ and $54 \%$ of the patients received short infusions in the second and third cycles, respectively. Although approximately one-third of the patients in the low-risk group experienced IRRs in the first rituximab cycle, the conversion rate to short infusion in the third cycle was over $80 \%$, and no 
patient experienced IRRs. Moreover, the overall incidence of IRRs to rituximab in the study participants was lower than those recorded in previous studies, where incidences of IRR to rituximab of up to $80 \%$ have been reported $[2,8]$. Hence, the present findings suggest that rituximab may be administered efficiently with a low incidence and severity of IRRs in patients with various B-NHL subtypes, provided a suitable protocol is used.

The incidences of IRRs were similar among the low-, moderate-, and high-risk groups. This may be due to a lower-than-expected incidence of IRRs in the moderate-risk group. In this group, we administered hydrocortisone phosphate, which may have reduced the incidence of IRRs. Nevertheless, the incidence of IRRs in the high-risk group was as high as we had anticipated. It is important to note, however, that the small number of patients in this group could have reduced the statistical power of the analysis.

In general, the incidence and severity of IRRs are highest after the initial rituximab cycle; hence, the first infusion takes approximately 4 to $6 \mathrm{~h}$. In contrast, IRRs to rituximab in subsequent cycles are infrequent and mostly manageable. For this reason, unless patients experience severe IRRs during the first rituximab administration, the second and subsequent doses are often administered by rapid infusion, requiring only 60 to 90 minutes in total [9]. Nevertheless, despite over a decade of clinical experience, no standardized method has yet been developed to determine which patients may safely receive rituximab by rapid infusion after completing conventional infusion procedures [9-11].

A pivotal phase III study evaluated the safety of a 90 min rituximab infusion in patients with previously untreated diffuse large B-cell and follicular lymphomas [12]. A total of 425 patients received the first rituximab cycle per the standard procedure, with a median infusion duration of $4 \mathrm{~h}$. The 363 patients who did not experience grade $\geq 3$ IRRs received subsequent infusions over 90 minutes. During cycle 2 (i.e., the first 90 min rituximab infusion), 135 (37\%) and 4 (1\%) patients experienced grade $1 / 2$ and 3 IRRs, respectively [12]. The present study did not evaluate the incidence of IRRs to rapid infusion of rituximab. However, it was assumed that our patients who received short infusions could safely receive rapid rituximab infusions because the maximum infusion rate of $400 \mathrm{mg} / \mathrm{h}$ in the short infusion cycle was not substantially different from that of the rapid infusion cycle. Therefore, our protocol may be applied to select patients for whom rapid infusion is considered safe.

Severe or life-threatening IRRs to rituximab, i.e., grade $\geq 3$, occur in approximately $10 \%$ of patients with B$\mathrm{NHL}[2,5,8]$. However, in the present study, none of the patients experienced IRRs to rituximab of grade 3 or higher. There are several possible reasons for the absence of severe IRRs. First, we avoided administering rituximab before the initial cytotoxic chemotherapy in $83 \%$ of the patients. This procedure may help reduce the tumor burden, which is the leading cause of IRR occurrence. Second, we limited the maximum infusion rate for the initial administration of rituximab to $100 \mathrm{mg} / \mathrm{h}$ in high-risk patients. Although this resulted in the total administration of rituximab requiring greater than $6 \mathrm{~h}$ in most patients, it helped prevent serious adverse reactions. Finally, we carefully chose patients who received rituximab by short infusion in the second cycle onwards, and this yielded a protocol compliance rate of $94 \%$. As a result, this study successfully minimized the severity of IRRs in B-NHL patients with various risk factors. 
One of the major limitations of this study is the lack of safety data on the rapid infusion of rituximab. This is partly because most patients in the study received biosimilar rituximab. While the incidence of IRRs is similar between biosimilar rituximab and reference rituximab [13], the package insert of biosimilar rituximab available in Japan does not support infusions within 60 to $90 \mathrm{~min}$. Furthermore, the number of patients in the high-risk group was small, resulting in statistically underpowered results. Therefore, the present findings should be validated in further multicenter studies.

\section{Conclusion}

Our step-by-step rituximab infusion protocol may allow for rituximab administration that is both safe and comfortable for patients and oncology practitioners. In addition, this novel protocol may help reduce the incidence of unexpected, severe adverse reactions to rituximab, which may require hospital admission, ultimately reducing the burden on healthcare providers and patients.

\section{Declarations}

Acknowledgments: The authors thank Mr. I. Odagiri, T. Yamauchi, Dr. H. Takahashi, Dr. M. Nakagawa, Dr. Y. Uchino, Dr. K. lizuka, Dr. T. Hamada, and Dr. N. Iriyama for their enormous contributions to this work.

Funding: This study was not funded by any third parties.

Availability of data and material: Anonymized data from this study are available upon legitimate request to the corresponding author.

Conflicts of interest: K. M. and Y. H. received speaker fees from Chugai and Kyowa-Kirin, which manufactures rituximab and its biosimilar products in Japan. The other authors have no conflicts of interest to disclose.

Authors' contributions: Conceptualization: D. T., T. H., and K. M; Methodology: D. T., T. H., A. U., and S. T.; Formal analysis and investigation: D. T., T. H., and K. M.; Supervision: S. O., Y. H., and Y. K.; writing original draft preparation: D. T. and K. M.; all authors critically revised the article and approved the final version to be published.

\section{References}

1. Salles G, Barrett M, Foà R, Maurer J, et al. Rituximab in B-Cell Hematologic Malignancies: A Review of 20 Years of Clinical Experience. Adv Ther. 2017;34:2232-73.

2. Paul F, Cartron G. Infusion-related reactions to rituximab: frequency, mechanisms and predictors. Expert Rev Clin Immunol. 2019;15:383-9.

3. Jung JW, Kang HR, Lee SH, et al. The incidence and risk factors of infusion-related reactions to rituximab for treating B cell malignancies in a single tertiary hospital. Oncologist. 2014;86:127-34. 
4. D'Arena G, Simeon V, Laurenti $L$, et al. Adverse drug reactions after intravenous rituximab infusion are more common in hematologic malignancies than in autoimmune disorders and can be predicted by the combination of few clinical and laboratory parameters: results from a retrospective, multicent. Leuk Lymphoma. 2017;58:2633-41.

5. Schwartzberg LS, Stepanski EJ, Fortner BV, et al. Retrospective chart review of severe infusion reactions with rituximab, cetuximab, and bevacizumab in community oncology practices: assessment of clinical consequences. Support Care Cancer. 2008;16:393-8.

6. Vogel HW. Infusion reactions: diagnosis, assessment, and management. Clin J Oncol Nurs. 2010;14:E10-21.

7. Hayama T, Miura K, Uchiike A, et al. A clinical prediction model for infusion-related reactions to rituximab in patients with B cell lymphomas. Int J Clin Pharm. 2017;39:380-5.

8. Chung $\mathrm{CH}$. Managing Premedications and the Risk for Reactions to Infusional Monoclonal Antibody Therapy. Oncologist. 2008;13:725-32.

9. Atmar J. Review of the safety and feasibility of rapid infusion of rituximab. J Oncol Pract. 2010;6:91-3.

10. Provencio M, Cerdeira S, Bonilla F, et al. Rapid-infusion rituximab in lymphoma treatment. Ann Oncol. 2006;17:1027-8.

11. Lang DS, Keefe DM, Schultz T, et al. Predictors of acute adverse events from rapid rituximab infusion. Support Care Cancer. 2013;21:2315-20.

12. Dakhil S, Hermann R, Schreeder MT, et al. Phase III safety study of rituximab administered as a 90minute infusion in patients with previously untreated diffuse large B-cell and follicular lymphoma. Leuk Lymphoma. 2014;55:2335-40.

13. Courville J, Nastoupil L, Kaila N, et al. Factors Influencing Infusion-Related Reactions Following Dosing of Reference Rituximab and PF-05280586, a Rituximab Biosimilar. BioDrugs. 2021;35:45968.

\section{Figures}




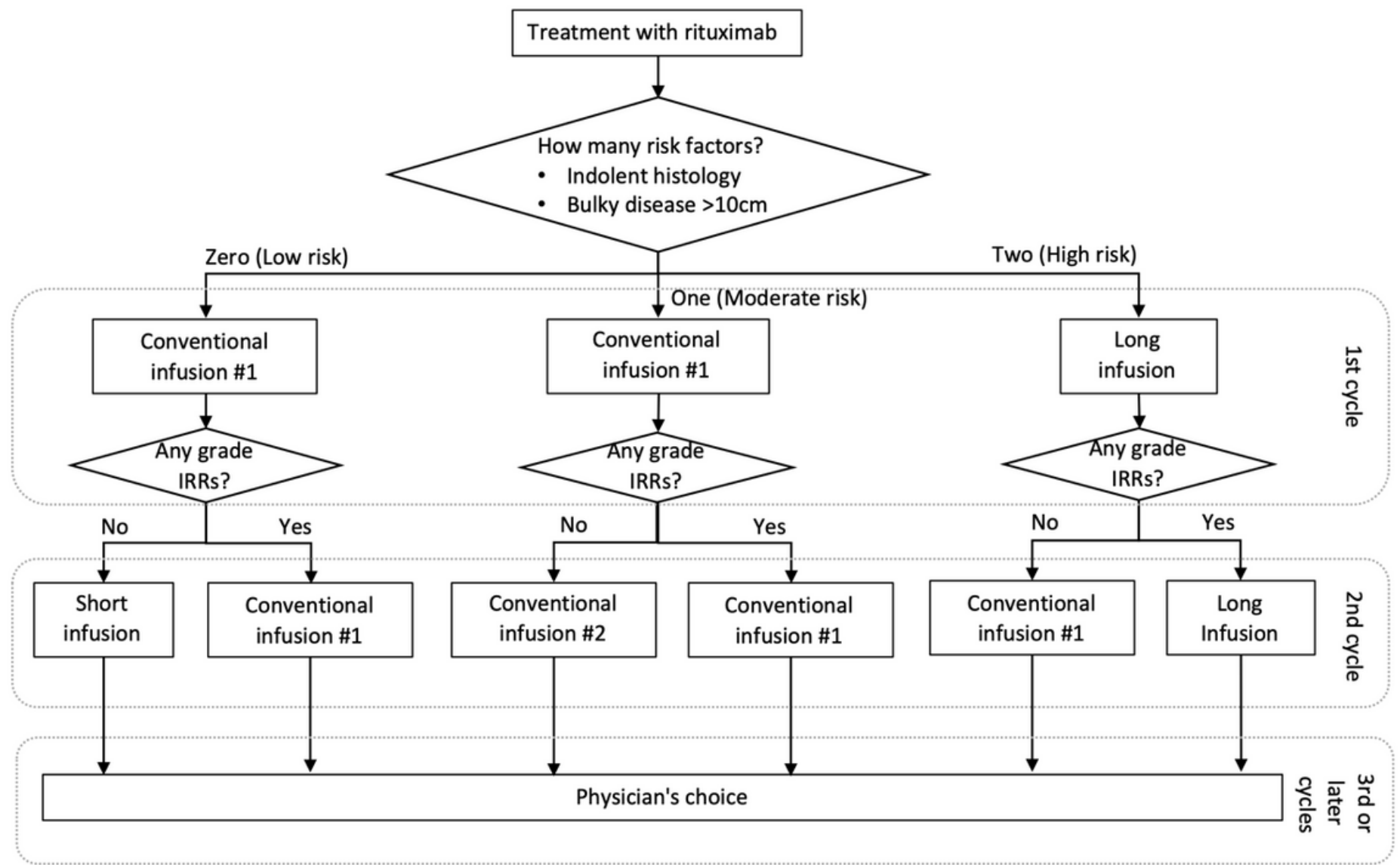

Figure 1

Flowchart of the study protocol. In conventional infusion \#1, the infusion rate of rituximab was set at 25 $\mathrm{mg} / \mathrm{h}$ for the first hour, $100 \mathrm{mg} / \mathrm{h}$ for the next hour, and a maximum of $200 \mathrm{mg} / \mathrm{h}$ thereafter. In long infusion, the infusion rate of rituximab was set at $25 \mathrm{mg} / \mathrm{h}$ for the first hour and a maximum of $100 \mathrm{mg} / \mathrm{h}$ thereafter. In short infusion, the infusion rate of rituximab was started at $100 \mathrm{mg} / \mathrm{h}$ for the first $30 \mathrm{~min}$ and increased by $100 \mathrm{mg} / \mathrm{h}$ every $30 \mathrm{~min}$ to a maximum of $400 \mathrm{mg} / \mathrm{h}$ thereafter. Finally, in conventional infusion \#2, the infusion rate of rituximab was set at $100 \mathrm{mg} / \mathrm{h}$ for the first hour and a maximum of 200 $\mathrm{mg} / \mathrm{h}$ thereafter 


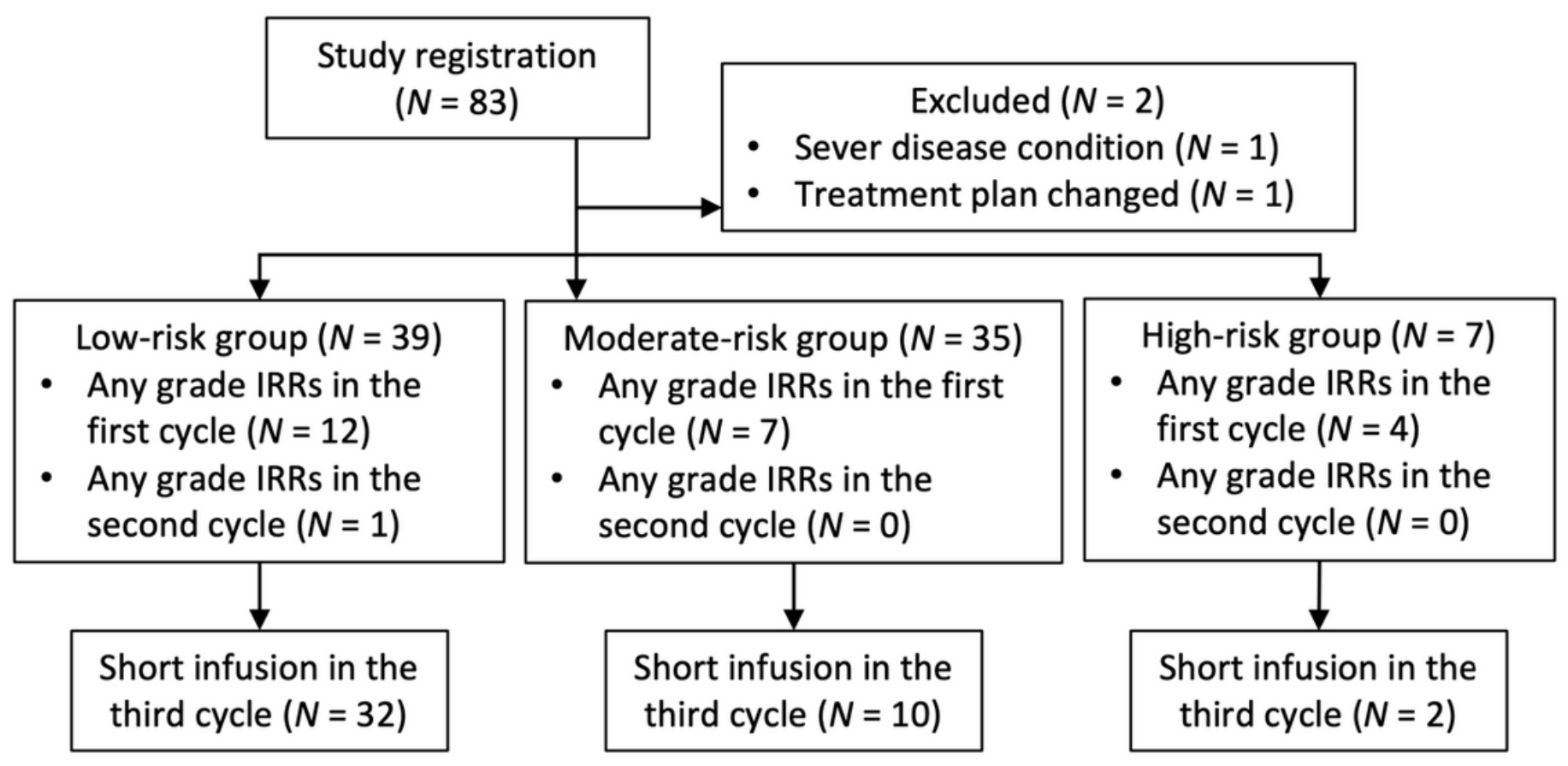

Figure 2

Flow diagram of the study results. Patients were stratified according to the number of relevant risk factors of infusion-related reactions (IRRs) due to rituximab (indolent histology and bulky disease) into low- (none), moderate- (one), or high-risk (two) groups. 


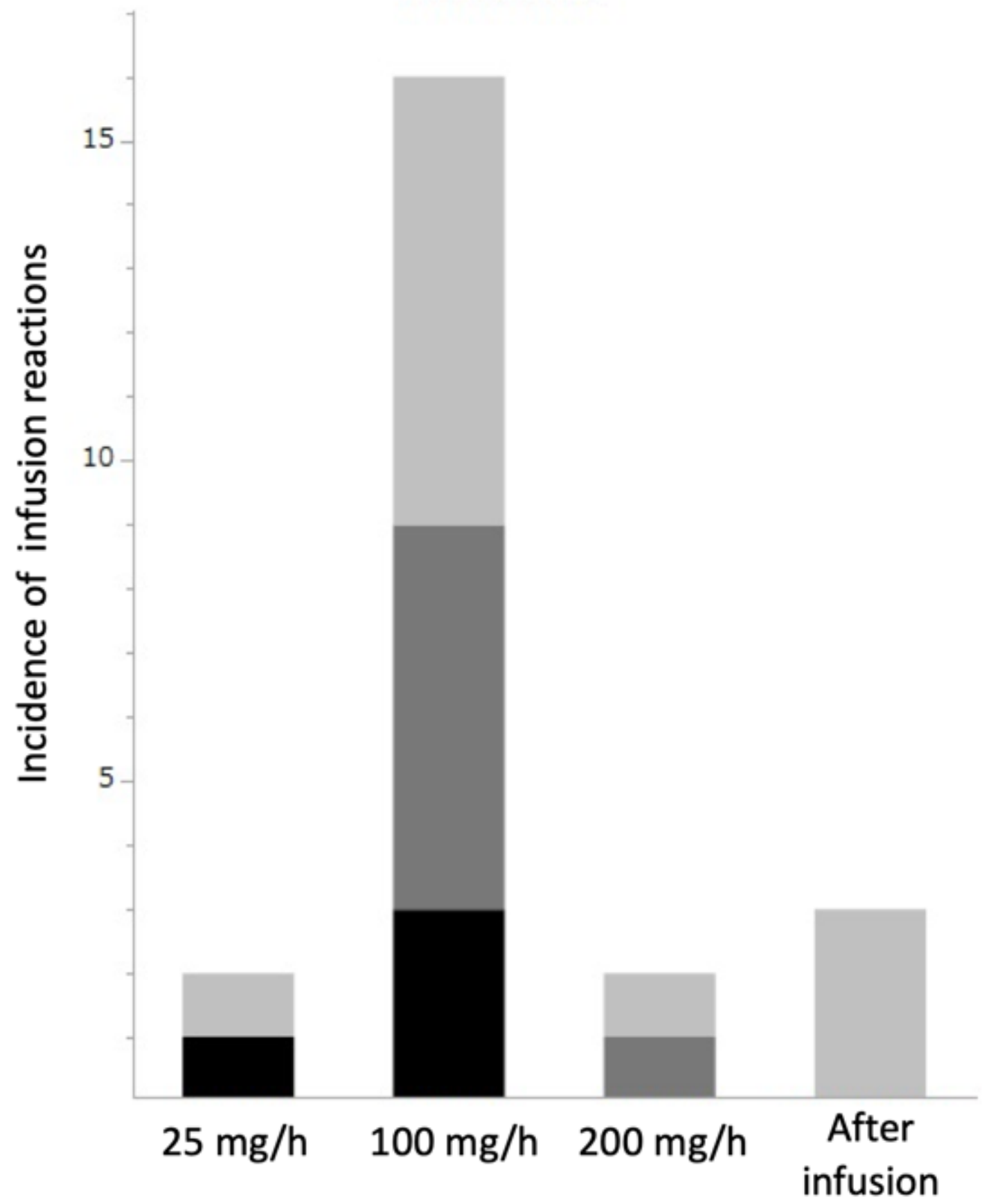

Figure 3

Incidence of infusion-related adverse reactions to rituximab and the infusion rates at the reaction onset in each risk group. The light gray, dark gray, and black bars represent the low-, moderate-, and high-risk groups, respectively 